\title{
APPENDIX 9
}

\section{Energy Conservation Estimates for Buildings in non-U.S. Countries}

To estimate the effects of trees on reducing energy use in buildings in cities outside the United States, i-Tree assigns some U.S. values to foreign locations based on average conditions within a U.S. climate region (based on McPherson and Simpson 1999; Fig. 15). The assignment of foreign locations to U.S. climate zones were based on, in order of priority:

1) User assignments through i-Tree database.

2) Foreign location similarities with U.S. latitude and coastal or interior geography.

3) Foreign location similarities based on average annual temperature.

Climate region assignments are given for cities in Australia (Table 23), provinces in Canada (Table 24), countries in Europe (Table 25), and cities in Mexico (Table 26).

The cost of electricity and fuels for Australia (2008) came from the Australian Energy Market Operator (2010); Canada (2011) from Natural Resources Canada (2012); Europe (2017) from Eurostat (2019) or, for the United Kingdom (2013) from Energy Solutions (2014); and Mexico (2016) from the International Energy Agency (2018).

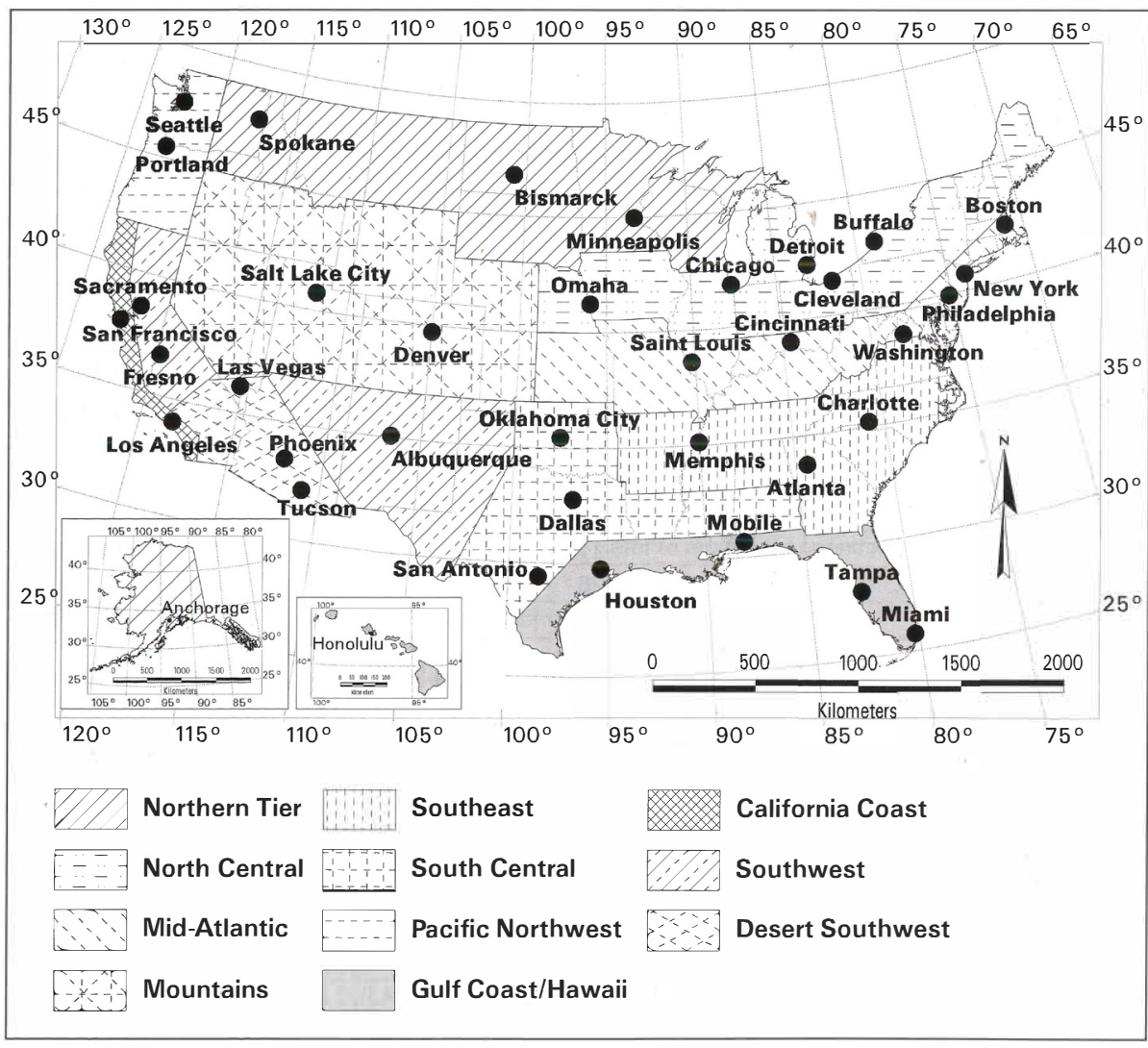

Figure 15.-Climate regions for the United States. Figure reprinted from McPherson and Simpson 1999.

Appendix 9 (https://doi.org/10.2737/NRS-GTR-200-2021-Appendix9) in: Nowak, David J. 2021. Understanding i-Tree: Summary of programs and methods. Gen. Tech. Rep. NRS-200-2021. Madison, WI: U.S. Department of Agriculture, Forest Service, Northern Research Station. 100 p. [plus 14 appendixes]. https://doi.org/10.2737/NRS-GTR-200-2021. 
Table 23.-U.S. climate regions assigned to municipalities in Australia

\begin{tabular}{|c|c|c|}
\hline State & Municipality & U.S. Climate Region \\
\hline Australian Capital Territory & Belconnen & Southeast \\
\hline Australian Capital Territory & Canberra Central & Southeast \\
\hline Australian Capital Territory & Gungahlin & Southeast \\
\hline Australian Capital Territory & Jerrabomberra & Southeast \\
\hline Australian Capital Territory & Majura & Southeast \\
\hline Australian Capital Territory & Molonglo & Southeast \\
\hline Australian Capital Territory & Tuggeranong & Southeast \\
\hline Australian Capital Territory & Weston Creek & Southeast \\
\hline Australian Capital Territory & Woden Valley & Southeast \\
\hline New South Wales & Albury City Council & South Central \\
\hline New South Wales & Armidale Dumaresq Council & South Central \\
\hline New South Wales & Ashfield Council & Gulf Coast/Hawaii \\
\hline New South Wales & Auburn Council & Gulf Coast/Hawaii \\
\hline New South Wales & Ballina Shire Council & Gulf Coast/Hawaii \\
\hline New South Wales & Balranald Shire Council & Desert Southwest \\
\hline New South Wales & Bankstown City Council & Gulf Coast/Hawaii \\
\hline New South Wales & Bathurst Regional Council & South Central \\
\hline New South Wales & Bega Valley Shire Council & South Central \\
\hline New South Wales & Bellingen Shire Council & Gulf Coast/Hawaii \\
\hline New South Wales & Berrigan Council & South Central \\
\hline New South Wales & Blacktown City Council & Gulf Coast/Hawaii \\
\hline New South Wales & Bland Shire Council & Desert Southwest \\
\hline New South Wales & Blayney Shire Council & South Central \\
\hline New South Wales & Blue Mountains City Council & South Central \\
\hline New South Wales & Bogan Shire Council & Desert Southwest \\
\hline New South Wales & Bombala Council & Southeast \\
\hline New South Wales & Boorowa Council & Southeast \\
\hline New South Wales & Botany Bay, The City of & Gulf Coast/Hawaii \\
\hline New South Wales & Bourke Shire Council & Desert Southwest \\
\hline New South Wales & Brewarrina Shire Council & Desert Southwest \\
\hline New South Wales & Broken Hill City Council & Desert Southwest \\
\hline New South Wales & Burwood Council & Gulf Coast/Hawaii \\
\hline New South Wales & Byron Shire Council & Gulf Coast/Hawaii \\
\hline New South Wales & Cabonne Shire Council & South Central \\
\hline New South Wales & Camden Council & Gulf Coast/Hawaii \\
\hline New South Wales & Campbelltown City Council & Gulf Coast/Hawaii \\
\hline New South Wales & Canada Bay Council, City of & Gulf Coast/Hawaii \\
\hline New South Wales & Canterbury City Council & Gulf Coast/Hawaii \\
\hline New South Wales & Carrathool Shire Council & Desert Southwest \\
\hline New South Wales & Central Darling Shire Council & Desert Southwest \\
\hline
\end{tabular}


Table 23.-Continued

\begin{tabular}{|c|c|c|}
\hline State & Municipality & U.S. Climate Region \\
\hline New South Wales & Cessnock City Council & South Central \\
\hline New South Wales & Clarence Valley Council & Gulf Coast/Hawaii \\
\hline New South Wales & Cobar Shire Council & Desert Southwest \\
\hline New South Wales & Coffs Harbour City Council & Gulf Coast/Hawaii \\
\hline New South Wales & Conargo Shire Council & South Central \\
\hline New South Wales & Coolamon Shire Council & Desert Southwest \\
\hline New South Wales & Cooma-Monaro Shire Council & Southeast \\
\hline New South Wales & Coonamble Shire Council & Desert Southwest \\
\hline New South Wales & Cootamundra Shire Council & South Central \\
\hline New South Wales & Corowa Shire Council & South Central \\
\hline New South Wales & Council of the City of Sydney & Gulf Coast/Hawaii \\
\hline New South Wales & Cowra Shire Council & South Central \\
\hline New South Wales & Deniliquin Council & South Central \\
\hline New South Wales & Dubbo City Council & South Central \\
\hline New South Wales & Dungog Shire Council & South Central \\
\hline New South Wales & Eurobodalla Shire Council & South Central \\
\hline New South Wales & Fairfield City Council & Gulf Coast/Hawaii \\
\hline New South Wales & Forbes Shire Council & Desert Southwest \\
\hline New South Wales & Gilgandra Shire Council & South Central \\
\hline New South Wales & Glen Innes Severn Council & South Central \\
\hline New South Wales & Gloucester Shire Council & South Central \\
\hline New South Wales & Gosford City Council & South Central \\
\hline New South Wales & Goulburn Mulwaree Council & South Central \\
\hline New South Wales & Great Lakes Council & Gulf Coast/Hawaii \\
\hline New South Wales & Greater Hume Shire Council & South Central \\
\hline New South Wales & Greater Taree City Council & Gulf Coast/Hawaii \\
\hline New South Wales & Griffith City Council & Desert Southwest \\
\hline New South Wales & Gundagai Shire Council & South Central \\
\hline New South Wales & Gunnedah Shire Council & Desert Southwest \\
\hline New South Wales & Guyra Shire Council & South Central \\
\hline New South Wales & Gwydir Shire Council & Gulf Coast/Hawaii \\
\hline New South Wales & Harden Shire Council & South Central \\
\hline New South Wales & Hawkesbury City Council & South Central \\
\hline New South Wales & Hay Shire Council & Desert Southwest \\
\hline New South Wales & Holroyd City Council & Gulf Coast/Hawaii \\
\hline New South Wales & Hornsby Shire Council & Gulf Coast/Hawaii \\
\hline New South Wales & Hunter's Hill Council & Gulf Coast/Hawaii \\
\hline New South Wales & Hurstville City Council & Gulf Coast/Hawaii \\
\hline New South Wales & Inverell Shire Council & Gulf Coast/Hawaii \\
\hline New South Wales & Jerilderie Shire Council & South Central \\
\hline
\end{tabular}


Table 23.-Continued

\begin{tabular}{|c|c|c|}
\hline State & Municipality & U.S. Climate Region \\
\hline New South Wales & Junee Shire Council & Desert Southwest \\
\hline New South Wales & Kempsey Shire Council & Gulf Coast/Hawaii \\
\hline New South Wales & Kiama Municipal Council & South Central \\
\hline New South Wales & Kogarah,City of & Gulf Coast/Hawaii \\
\hline New South Wales & Ku-ring-gai Council & Gulf Coast/Hawaii \\
\hline New South Wales & Kyogle Council & Gulf Coast/Hawaii \\
\hline New South Wales & Lachlan Shire Council & Desert Southwest \\
\hline New South Wales & Lake Macquarie City Council & Gulf Coast/Hawaii \\
\hline New South Wales & Lane Cove Municipal Council & Gulf Coast/Hawaii \\
\hline New South Wales & Leeton Shire Council & Desert Southwest \\
\hline New South Wales & Leichhardt Municipal Council & Gulf Coast/Hawaii \\
\hline New South Wales & Lismore City Council & Gulf Coast/Hawaii \\
\hline New South Wales & Lithgow Council, City of & South Central \\
\hline New South Wales & Liverpool City Council & Gulf Coast/Hawaii \\
\hline New South Wales & Liverpool Plains Shire Council & Gulf Coast/Hawaii \\
\hline New South Wales & Lockhart Shire Council & Desert Southwest \\
\hline New South Wales & Maitland City Council & South Central \\
\hline New South Wales & Manly Council & Gulf Coast/Hawaii \\
\hline New South Wales & Marrickville Council & Gulf Coast/Hawaii \\
\hline New South Wales & Mid-Western Regional Council & South Central \\
\hline New South Wales & Moree Plains Shire Council & Desert Southwest \\
\hline New South Wales & Mosman Municipal Council & Gulf Coast/Hawaii \\
\hline New South Wales & Murray Shire Council & South Central \\
\hline New South Wales & Murrumbidgee Shire Council & Desert Southwest \\
\hline New South Wales & Muswellbrook Shire Council & South Central \\
\hline New South Wales & Nambucca Shire Council & Gulf Coast/Hawaii \\
\hline New South Wales & Narrabri Shire Council & Desert Southwest \\
\hline New South Wales & Narrandera Shire Council & Desert Southwest \\
\hline New South Wales & Narromine Shire Council & Desert Southwest \\
\hline New South Wales & Newcastle City Council & Gulf Coast/Hawaii \\
\hline New South Wales & North Sydney Council & Gulf Coast/Hawaii \\
\hline New South Wales & Oberon Council & South Central \\
\hline New South Wales & Orange City Council & South Central \\
\hline New South Wales & Palerang Council & South Central \\
\hline New South Wales & Parkes Shire Council & Desert Southwest \\
\hline New South Wales & Parramatta City Council & Gulf Coast/Hawaii \\
\hline New South Wales & Penrith City Council & Gulf Coast/Hawaii \\
\hline New South Wales & Pittwater Council & Gulf Coast/Hawaii \\
\hline New South Wales & Port Macquarie-Hastings Council & Gulf Coast/Hawaii \\
\hline New South Wales & Port Stephens Council & Gulf Coast/Hawaii \\
\hline
\end{tabular}


Table 23.-Continued

\begin{tabular}{|c|c|c|}
\hline State & Municipality & U.S. Climate Region \\
\hline New South Wales & Queanbeyan City Council & Southeast \\
\hline New South Wales & Randwick City Council & Gulf Coast/Hawaii \\
\hline New South Wales & Richmond Valley Council & Gulf Coast/Hawaii \\
\hline New South Wales & Rockdale City Council & Gulf Coast/Hawaii \\
\hline New South Wales & Ryde City Council & Gulf Coast/Hawaii \\
\hline New South Wales & Shellharbour City Council & South Central \\
\hline New South Wales & Shoalhaven City Council & South Central \\
\hline New South Wales & Singleton Shire Council & South Central \\
\hline New South Wales & Snowy River Shire Council & Southeast \\
\hline New South Wales & Strathfield Municipal Council & Gulf Coast/Hawaii \\
\hline New South Wales & Sutherland Shire Council & Gulf Coast/Hawaii \\
\hline New South Wales & Tamworth Regional Council & Gulf Coast/Hawaii \\
\hline New South Wales & Temora Shire Council & Desert Southwest \\
\hline New South Wales & Tenterfield Shire Council & South Central \\
\hline New South Wales & The Hills Shire Council & Gulf Coast/Hawaii \\
\hline New South Wales & Tumbarumba Shire Council & South Central \\
\hline New South Wales & Tumut Shire Council & Southeast \\
\hline New South Wales & Tweed Shire Council & Gulf Coast/Hawaii \\
\hline New South Wales & Upper Hunter Shire Council & South Central \\
\hline New South Wales & Upper Lachlan Shire Council & Southeast \\
\hline New South Wales & Uralla Shire Council & South Central \\
\hline New South Wales & Urana Shire Council & South Central \\
\hline New South Wales & Wagga Wagga City Council & Desert Southwest \\
\hline New South Wales & Wakool Shire Council & South Central \\
\hline New South Wales & Walcha Council & South Central \\
\hline New South Wales & Walgett Shire Council & Desert Southwest \\
\hline New South Wales & Warren Shire Council & Desert Southwest \\
\hline New South Wales & Warringah Council & Gulf Coast/Hawaii \\
\hline New South Wales & Warrumbungle Shire Council & South Central \\
\hline New South Wales & Waverley Council & Gulf Coast/Hawaii \\
\hline New South Wales & Weddin Council & Desert Southwest \\
\hline New South Wales & Wellington Council & South Central \\
\hline New South Wales & Wentworth Shire Council & Desert Southwest \\
\hline New South Wales & Willoughby City Council & Gulf Coast/Hawaii \\
\hline New South Wales & Wingecarribee Shire Council & South Central \\
\hline New South Wales & Wollondilly Shire Council & South Central \\
\hline New South Wales & Wollongong City Council & South Central \\
\hline New South Wales & Woollahra Municipal Council & Gulf Coast/Hawaii \\
\hline New South Wales & Wyong Shire Council & South Central \\
\hline New South Wales & Yass Valley Council & Southeast \\
\hline
\end{tabular}


Table 23.-Continued

\begin{tabular}{|c|c|c|}
\hline State & Municipality & U.S. Climate Region \\
\hline New South Wales & Young Shire Council & South Central \\
\hline Northern Territory & Alice Springs Town Council & Desert Southwest \\
\hline Northern Territory & Barkly Shire Council & Gulf Coast/Hawaii \\
\hline Northern Territory & Central Desert Shire Council & Desert Southwest \\
\hline Northern Territory & Coomalie Community Govt Council & Gulf Coast/Hawaii \\
\hline Northern Territory & Darwin City Council & Gulf Coast/Hawaii \\
\hline Northern Territory & East Arnhem Shire Council & Gulf Coast/Hawaii \\
\hline Northern Territory & Katherine Town Council & Gulf Coast/Hawaii \\
\hline Northern Territory & Litchfield Shire Council & Gulf Coast/Hawaii \\
\hline Northern Territory & Macdonnell Shire Council & Desert Southwest \\
\hline Northern Territory & Palmerston City Council & Gulf Coast/Hawaii \\
\hline Northern Territory & Roper Gulf Shire Council & Gulf Coast/Hawaii \\
\hline Northern Territory & Tiwi Islands Local Government & Gulf Coast/Hawaii \\
\hline Northern Territory & Victoria Daly Shire Council & Gulf Coast/Hawaii \\
\hline Northern Territory & Wagait Shire Council & Gulf Coast/Hawaii \\
\hline Northern Territory & West Arnhem Shire Council & Gulf Coast/Hawaii \\
\hline Queensland & Aurukun Shire Council & Gulf Coast/Hawaii \\
\hline Queensland & Balonne Shire Council & Desert Southwest \\
\hline Queensland & Banana Shire Council & Gulf Coast/Hawaii \\
\hline Queensland & Barcaldine Regional Council & Desert Southwest \\
\hline Queensland & Barcoo Shire Council & Desert Southwest \\
\hline Queensland & Blackall-Tambo Regional Council & Desert Southwest \\
\hline Queensland & Boulia Shire Council & Desert Southwest \\
\hline Queensland & Brisbane City Council & Gulf Coast/Hawaii \\
\hline Queensland & Bulloo Shire Council & Desert Southwest \\
\hline Queensland & Bundaberg Regional Council & Gulf Coast/Hawaii \\
\hline Queensland & Burdekin Shire Council & Gulf Coast/Hawaii \\
\hline Queensland & Burke Shire Council & Gulf Coast/Hawaii \\
\hline Queensland & Cairns Regional Council & Gulf Coast/Hawaii \\
\hline Queensland & Carpentaria Shire Council & Gulf Coast/Hawaii \\
\hline Queensland & Cassowary Coast Regional Council & Gulf Coast/Hawaii \\
\hline Queensland & Central Highlands Regional Council & Gulf Coast/Hawaii \\
\hline Queensland & Charters Towers Regional Council & Gulf Coast/Hawaii \\
\hline Queensland & Cherbourg Aboriginal Shire Council & Gulf Coast/Hawaii \\
\hline Queensland & Cloncurry Shire Council & Desert Southwest \\
\hline Queensland & Cook Shire Council & Gulf Coast/Hawaii \\
\hline Queensland & Croydon Shire Council & Gulf Coast/Hawaii \\
\hline Queensland & Diamantina Shire Council & Desert Southwest \\
\hline Queensland & Doomadgee Aboriginal Shire Council & Gulf Coast/Hawaii \\
\hline Queensland & Etheridge Shire Council & Gulf Coast/Hawaii \\
\hline
\end{tabular}


Table 23.-Continued

\begin{tabular}{|c|c|c|}
\hline State & Municipality & U.S. Climate Region \\
\hline Queensland & Flinders Shire Council & Desert Southwest \\
\hline Queensland & Fraser Coast Regional Council & Gulf Coast/Hawaii \\
\hline Queensland & Gladstone Regional Council & Gulf Coast/Hawaii \\
\hline Queensland & Gold Coast City Council & Gulf Coast/Hawaii \\
\hline Queensland & Goondiwindi Regional Council & California Coast \\
\hline Queensland & Gympie Regional Council & Gulf Coast/Hawaii \\
\hline Queensland & Hinchinbrook Shire Council & Gulf Coast/Hawaii \\
\hline Queensland & Hope Vale Aboriginal Shire Council & Gulf Coast/Hawaii \\
\hline Queensland & Ipswich City Council & Gulf Coast/Hawaii \\
\hline Queensland & Isaac Regional Council & Gulf Coast/Hawaii \\
\hline Queensland & Kowanyama Aboriginal Shire Council & Gulf Coast/Hawaii \\
\hline Queensland & Lockhart River Aboriginal Shire Council & Gulf Coast/Hawaii \\
\hline Queensland & Lockyer Valley Regional Council & Gulf Coast/Hawaii \\
\hline Queensland & Logan City Council & Gulf Coast/Hawaii \\
\hline Queensland & Longreach Regional Council & Desert Southwest \\
\hline Queensland & Mackay Regional Council & Gulf Coast/Hawaii \\
\hline Queensland & Mapoon Aboriginal Shire Council & Gulf Coast/Hawaii \\
\hline Queensland & Maranoa Regional Council & Desert Southwest \\
\hline Queensland & McKinlay Shire Council & Desert Southwest \\
\hline Queensland & Moreton Bay Regional Council & Gulf Coast/Hawaii \\
\hline Queensland & Mornington Shire Council & Gulf Coast/Hawaii \\
\hline Queensland & Mount Isa City Council & Desert Southwest \\
\hline Queensland & Murweh Shire Council & Desert Southwest \\
\hline Queensland & Napranum Aboriginal Shire Council & Gulf Coast/Hawaii \\
\hline Queensland & North Burnett Regional Council & Gulf Coast/Hawaii \\
\hline Queensland & Northern Peninsula Area Regional Council & Gulf Coast/Hawaii \\
\hline Queensland & Palm Island Aboriginal Shire Council & Gulf Coast/Hawaii \\
\hline Queensland & Paroo Shire Council & Desert Southwest \\
\hline Queensland & Pormpuraaw Aboriginal Shire Council & Gulf Coast/Hawaii \\
\hline Queensland & Quilpie Shire Council & Desert Southwest \\
\hline Queensland & Redland City Council & Gulf Coast/Hawaii \\
\hline Queensland & Richmond Shire Council & Desert Southwest \\
\hline Queensland & Rockhampton Regional Council & Gulf Coast/Hawaii \\
\hline Queensland & Scenic Rim Regional Council & South Central \\
\hline Queensland & Somerset Regional Council & Gulf Coast/Hawaii \\
\hline Queensland & South Burnett Regional Council & Gulf Coast/Hawaii \\
\hline Queensland & Southern Downs Regional Council & Gulf Coast/Hawaii \\
\hline Queensland & Sunshine Coast Regional Council & Gulf Coast/Hawaii \\
\hline Queensland & Tablelands Regional Council & Gulf Coast/Hawaii \\
\hline Queensland & Toowoomba Regional Council & Gulf Coast/Hawaii \\
\hline
\end{tabular}


Table 23.-Continued

\begin{tabular}{|c|c|c|}
\hline State & Municipality & U.S. Climate Region \\
\hline Queensland & Torres Shire Council & Gulf Coast/Hawaii \\
\hline Queensland & Torres Strait Island Regional Council & Gulf Coast/Hawaii \\
\hline Queensland & Townsville City Council & Gulf Coast/Hawaii \\
\hline Queensland & Weipa Town Council & Gulf Coast/Hawaii \\
\hline Queensland & Western Downs Regional Council & Gulf Coast/Hawaii \\
\hline Queensland & Whitsunday Regional Council & Gulf Coast/Hawaii \\
\hline Queensland & Winton Shire Council & Desert Southwest \\
\hline Queensland & Woorabinda Aboriginal Shire Council & Gulf Coast/Hawaii \\
\hline Queensland & Wujal Wujal Aboriginal Shire Council & Gulf Coast/Hawaii \\
\hline Queensland & Yarrabah Aboriginal Shire Council & Gulf Coast/Hawaii \\
\hline South Australia & Adelaide City Council & Desert Southwest \\
\hline South Australia & Adelaide Hills Council & Desert Southwest \\
\hline South Australia & Alexandrina Council & Desert Southwest \\
\hline South Australia & Anangu Pitjantjatjara Yankunytjatjara & Desert Southwest \\
\hline South Australia & Barossa Council, The & Desert Southwest \\
\hline South Australia & Barunga West, District Council of & Desert Southwest \\
\hline South Australia & Berri Barmera Council & Desert Southwest \\
\hline South Australia & Burnside, The City of & Desert Southwest \\
\hline South Australia & Campbelltown City Council & Desert Southwest \\
\hline South Australia & Ceduna, District Council of & Desert Southwest \\
\hline South Australia & Charles Sturt, City of & Desert Southwest \\
\hline South Australia & Clare \& Gilbert Valleys Council & Desert Southwest \\
\hline South Australia & Cleve, District Council of & Desert Southwest \\
\hline South Australia & Coober Pedy, District Council of & Desert Southwest \\
\hline South Australia & Coorong District Council, The & Desert Southwest \\
\hline South Australia & Copper Coast, District Council of & Desert Southwest \\
\hline South Australia & Elliston, District Council of & Desert Southwest \\
\hline South Australia & Flinders Ranges Council, The & Desert Southwest \\
\hline South Australia & Franklin Harbour, District Council of & Desert Southwest \\
\hline South Australia & Gawler, Town of & Desert Southwest \\
\hline South Australia & Gerard Community Council & Desert Southwest \\
\hline South Australia & Goyder, Regional Council of & Desert Southwest \\
\hline South Australia & Grant, District Council of & Desert Southwest \\
\hline South Australia & Holdfast Bay, City of & Desert Southwest \\
\hline South Australia & Kangaroo Island Council & Desert Southwest \\
\hline South Australia & Karoonda East Murray, District Council of & Desert Southwest \\
\hline South Australia & Kimba, District Council of & Desert Southwest \\
\hline South Australia & Kingston District Council & Desert Southwest \\
\hline South Australia & Light Regional Council & Desert Southwest \\
\hline South Australia & Lower Eyre Peninsula, District Council & Desert Southwest \\
\hline
\end{tabular}


Table 23.-Continued

\begin{tabular}{|c|c|c|}
\hline State & Municipality & U.S. Climate Region \\
\hline South Australia & Loxton Waikerie, District Council of & Desert Southwest \\
\hline South Australia & Mallala, District Council of & Desert Southwest \\
\hline South Australia & Maralinga Tjarutja & Desert Southwest \\
\hline South Australia & Marion, City of & Desert Southwest \\
\hline South Australia & Mid Murray Council & Desert Southwest \\
\hline South Australia & Mitcham, City of & Desert Southwest \\
\hline South Australia & Mt Barker, District Council of & Desert Southwest \\
\hline South Australia & Mt Gambier, City of & Desert Southwest \\
\hline South Australia & Mt Remarkable, District Council of & Desert Southwest \\
\hline South Australia & Murray Bridge, The Rural City of & Desert Southwest \\
\hline South Australia & Naracoorte Lucindale Council & Desert Southwest \\
\hline South Australia & Nepabunna Community Incorporated & Desert Southwest \\
\hline South Australia & Northern Areas Council & Desert Southwest \\
\hline South Australia & Onkaparinga, City of & Desert Southwest \\
\hline South Australia & Orroroo Carrieton, District Council of & Desert Southwest \\
\hline South Australia & Outback Areas Community Development Trust & Desert Southwest \\
\hline South Australia & Payneham \& St Peters, The City Of Norwood, & Desert Southwest \\
\hline South Australia & Peterborough, District Council of & Desert Southwest \\
\hline South Australia & Playford, City of & Desert Southwest \\
\hline South Australia & Port Adelaide Enfield, City of & Desert Southwest \\
\hline South Australia & Port Augusta City Council & Desert Southwest \\
\hline South Australia & Port Lincoln, City of & Desert Southwest \\
\hline South Australia & Port Pirie Regional Council & Desert Southwest \\
\hline South Australia & Prospect, City of & Desert Southwest \\
\hline South Australia & Renmark Paringa, District Council of & Desert Southwest \\
\hline South Australia & Robe, District Council of & Desert Southwest \\
\hline South Australia & Roxby Downs, Municipal Council of & Desert Southwest \\
\hline South Australia & Salisbury, City of & Desert Southwest \\
\hline South Australia & Southern Mallee District Council & Desert Southwest \\
\hline South Australia & Streaky Bay, District Council of & Desert Southwest \\
\hline South Australia & Tatiara District Council & Desert Southwest \\
\hline South Australia & Tea Tree Gully, City of & Desert Southwest \\
\hline South Australia & Tumby Bay, District Council of & Desert Southwest \\
\hline South Australia & Unley, City of & Desert Southwest \\
\hline South Australia & Victor Harbor, City of & Desert Southwest \\
\hline South Australia & Wakefield Regional Council & Desert Southwest \\
\hline South Australia & Walkerville, Corporation of the Town of & Desert Southwest \\
\hline South Australia & Wattle Range Council & Desert Southwest \\
\hline South Australia & West Torrens, City of & Desert Southwest \\
\hline South Australia & Whyalla, The Corporation of the City of & Desert Southwest \\
\hline
\end{tabular}


Table 23.-Continued

\begin{tabular}{|c|c|c|}
\hline State & Municipality & U.S. Climate Region \\
\hline South Australia & Wudinna District Council & Desert Southwest \\
\hline South Australia & Yalata & Desert Southwest \\
\hline South Australia & Yankalilla, District Council of & Desert Southwest \\
\hline South Australia & Yorke Peninsula, District Council of & Desert Southwest \\
\hline Tasmania & Break O'Day Council & Pacific Northwest \\
\hline Tasmania & Brighton Council & Pacific Northwest \\
\hline Tasmania & Burnie City Council & Pacific Northwest \\
\hline Tasmania & Central Coast Council & Pacific Northwest \\
\hline Tasmania & Central Highlands Council & Pacific Northwest \\
\hline Tasmania & Circular Head Council & Pacific Northwest \\
\hline Tasmania & Clarence City Council & Pacific Northwest \\
\hline Tasmania & Derwent Valley Council & Pacific Northwest \\
\hline Tasmania & Devonport City Council & Pacific Northwest \\
\hline Tasmania & Dorset Council & Pacific Northwest \\
\hline Tasmania & Flinders Council & Pacific Northwest \\
\hline Tasmania & George Town Council & Pacific Northwest \\
\hline Tasmania & Glamorgan Spring Bay Council & Pacific Northwest \\
\hline Tasmania & Glenorchy City Council & Pacific Northwest \\
\hline Tasmania & Hobart City Council & Pacific Northwest \\
\hline Tasmania & Huon Valley Council & Pacific Northwest \\
\hline Tasmania & Kentish Council & Pacific Northwest \\
\hline Tasmania & King Island Council & Pacific Northwest \\
\hline Tasmania & Kingborough Council & Pacific Northwest \\
\hline Tasmania & Latrobe Council & Pacific Northwest \\
\hline Tasmania & Launceston City Council & Pacific Northwest \\
\hline Tasmania & Meander Valley Council & Pacific Northwest \\
\hline Tasmania & Northern Midlands Council & Pacific Northwest \\
\hline Tasmania & Sorell Council & Pacific Northwest \\
\hline Tasmania & Southern Midlands Council & Pacific Northwest \\
\hline Tasmania & Tasman Council & Pacific Northwest \\
\hline Tasmania & Waratah - Wynyard Council & Pacific Northwest \\
\hline Tasmania & West Coast Council & Pacific Northwest \\
\hline Tasmania & West Tamar Council & Pacific Northwest \\
\hline Victoria & Alpine Shire Council & California Coast \\
\hline Victoria & Ararat Rural City Council & California Coast \\
\hline Victoria & Ballarat City Council & California Coast \\
\hline Victoria & Banyule City Council & California Coast \\
\hline Victoria & Bass Coast Shire Council & California Coast \\
\hline Victoria & Baw Baw Shire Council & California Coast \\
\hline Victoria & Bayside City Council & California Coast \\
\hline
\end{tabular}


Table 23.-Continued

\begin{tabular}{|c|c|c|}
\hline State & Municipality & U.S. Climate Region \\
\hline Victoria & Benalla Rural City Council & California Coast \\
\hline Victoria & Boroondara City Council & California Coast \\
\hline Victoria & Borough of Queenscliffe & California Coast \\
\hline Victoria & Brimbank City Council & California Coast \\
\hline Victoria & Buloke Shire Council & California Coast \\
\hline Victoria & Campaspe Shire Council & California Coast \\
\hline Victoria & Cardinia Shire Council & California Coast \\
\hline Victoria & Casey City Council & California Coast \\
\hline Victoria & Central Goldfields Shire Council & California Coast \\
\hline Victoria & Colac Otway Shire Council & California Coast \\
\hline Victoria & Corangamite Shire Council & California Coast \\
\hline Victoria & Darebin City Council & California Coast \\
\hline Victoria & East Gippsland Shire Council & California Coast \\
\hline Victoria & Frankston City Council & California Coast \\
\hline Victoria & Gannawarra Shire Council & Desert Southwest \\
\hline Victoria & Glen Eira City Council & California Coast \\
\hline Victoria & Glenelg Shire Council & California Coast \\
\hline Victoria & Golden Plains Shire Council & California Coast \\
\hline Victoria & Greater Bendigo City Council & California Coast \\
\hline Victoria & Greater Dandenong City Council & California Coast \\
\hline Victoria & Greater Geelong City Council & California Coast \\
\hline Victoria & Greater Shepparton City Council & California Coast \\
\hline Victoria & Hepburn Shire Council & California Coast \\
\hline Victoria & Hindmarsh Shire Council & Desert Southwest \\
\hline Victoria & Hobsons Bay City Council & California Coast \\
\hline Victoria & Horsham Rural City Council & California Coast \\
\hline Victoria & Hume City Council & California Coast \\
\hline Victoria & Indigo Shire Council & California Coast \\
\hline Victoria & Kingston City Council & California Coast \\
\hline Victoria & Knox City Council & California Coast \\
\hline Victoria & Latrobe City Council & California Coast \\
\hline Victoria & Loddon Shire Council & Desert Southwest \\
\hline Victoria & Macedon Ranges Shire Council & California Coast \\
\hline Victoria & Manningham City Council & California Coast \\
\hline Victoria & Mansfield Shire Council & California Coast \\
\hline Victoria & Maribyrnong City Council & California Coast \\
\hline Victoria & Maroondah City Council & California Coast \\
\hline Victoria & Melbourne City Council & California Coast \\
\hline Victoria & Melton Shire Council & California Coast \\
\hline Victoria & Mildura Rural City Council & Desert Southwest \\
\hline
\end{tabular}


Table 23.-Continued

\begin{tabular}{|c|c|c|}
\hline State & Municipality & U.S. Climate Region \\
\hline Victoria & Mitchell Shire Council & California Coast \\
\hline Victoria & Moira Shire Council & California Coast \\
\hline Victoria & Monash City Council & California Coast \\
\hline Victoria & Moonee Valley City Council & California Coast \\
\hline Victoria & Moorabool Shire Council & California Coast \\
\hline Victoria & Moreland City Council & California Coast \\
\hline Victoria & Mornington Peninsula Shire Council & California Coast \\
\hline Victoria & Mount Alexander Shire Council & California Coast \\
\hline Victoria & Moyne Shire Council & California Coast \\
\hline Victoria & Murrindindi Shire Council & California Coast \\
\hline Victoria & Nillumbik Shire Council & California Coast \\
\hline Victoria & Northern Grampians Shire Council & California Coast \\
\hline Victoria & Port Phillip City Council & California Coast \\
\hline Victoria & Pyrenees Shire Council & California Coast \\
\hline Victoria & South Gippsland Shire Council & California Coast \\
\hline Victoria & Southern Grampians Shire Council & California Coast \\
\hline Victoria & Stonnington City Council & California Coast \\
\hline Victoria & Strathbogie Shire Council & California Coast \\
\hline Victoria & Surf Coast Shire Council & California Coast \\
\hline Victoria & Swan Hill Rural City Council & Desert Southwest \\
\hline Victoria & Towong Shire Council & California Coast \\
\hline Victoria & Wangaratta Rural City Council & California Coast \\
\hline Victoria & Warrnambool City Council & California Coast \\
\hline Victoria & Wellington Shire Council & California Coast \\
\hline Victoria & West Wimmera Shire Council & California Coast \\
\hline Victoria & Whitehorse City Council & California Coast \\
\hline Victoria & Whittlesea City Council & California Coast \\
\hline Victoria & Wodonga City Council & California Coast \\
\hline Victoria & Wyndham City Council & California Coast \\
\hline Victoria & Yarra City Council & California Coast \\
\hline Victoria & Yarra Ranges Shire Council & California Coast \\
\hline Victoria & Yarriambiack Shire Council & Desert Southwest \\
\hline Western Australia & Albany, City of & California Coast \\
\hline Western Australia & Armadale, City of & Desert Southwest \\
\hline Western Australia & Ashburton, Shire of & Gulf Coast/Hawaii \\
\hline Western Australia & Augusta-Margaret River, Shire of & Desert Southwest \\
\hline Western Australia & Bassendean, Town of & Desert Southwest \\
\hline Western Australia & Bayswater, City of & Desert Southwest \\
\hline Western Australia & Belmont, City of & Desert Southwest \\
\hline Western Australia & Beverley, Shire of & Desert Southwest \\
\hline
\end{tabular}


Table 23.-Continued

\begin{tabular}{|c|c|c|}
\hline State & Municipality & U.S. Climate Region \\
\hline Western Australia & Boddington, Shire of & Desert Southwest \\
\hline Western Australia & Boyup Brook, Shire of & Desert Southwest \\
\hline Western Australia & Bridgetown-Greenbushes, Shire of & Desert Southwest \\
\hline Western Australia & Brookton, Shire of & Desert Southwest \\
\hline Western Australia & Broome, Shire of & Gulf Coast/Hawaii \\
\hline Western Australia & Broomehill-Tambellup, Shire of & Desert Southwest \\
\hline Western Australia & Bruce Rock, Shire of & Desert Southwest \\
\hline Western Australia & Bunbury, City of & Desert Southwest \\
\hline Western Australia & Busselton, Shire of & Desert Southwest \\
\hline Western Australia & Cambridge, Town of & Desert Southwest \\
\hline Western Australia & Canning, City of & Desert Southwest \\
\hline Western Australia & Capel, Shire of & Desert Southwest \\
\hline Western Australia & Carnamah, Shire of & Desert Southwest \\
\hline Western Australia & Carnarvon, Shire of & Gulf Coast/Hawaii \\
\hline Western Australia & Chapman Valley, Shire of & Desert Southwest \\
\hline Western Australia & Chittering, Shire of & Desert Southwest \\
\hline Western Australia & Christmas Island, Shire of & Gulf Coast/Hawaii \\
\hline Western Australia & Claremont, Town of & Desert Southwest \\
\hline Western Australia & Cockburn, City of & Desert Southwest \\
\hline Western Australia & Cocos (Keeling) Islands, Shire of & Gulf Coast/Hawaii \\
\hline Western Australia & Collie, Shire of & Desert Southwest \\
\hline Western Australia & Coolgardie, Shire of & Desert Southwest \\
\hline Western Australia & Coorow, Shire of & Desert Southwest \\
\hline Western Australia & Corrigin, Shire of & Desert Southwest \\
\hline Western Australia & Cottesloe, Town of & Desert Southwest \\
\hline Western Australia & Cranbrook, Shire of & Desert Southwest \\
\hline Western Australia & Cuballing, Shire of & Desert Southwest \\
\hline Western Australia & Cue, Shire of & Desert Southwest \\
\hline Western Australia & Cunderdin, Shire of & Desert Southwest \\
\hline Western Australia & Dalwallinu, Shire of & Desert Southwest \\
\hline Western Australia & Dandaragan, Shire of & Desert Southwest \\
\hline Western Australia & Dardanup, Shire of & Desert Southwest \\
\hline Western Australia & Denmark, Shire of & California Coast \\
\hline Western Australia & Derby-West Kimberley, Shire of & Gulf Coast/Hawaii \\
\hline Western Australia & Donnybrook-Balingup, Shire of & Desert Southwest \\
\hline Western Australia & Dowerin, Shire of & Desert Southwest \\
\hline Western Australia & Dumbleyung, Shire of & Desert Southwest \\
\hline Western Australia & Dundas, Shire of & Desert Southwest \\
\hline Western Australia & East Fremantle, Town of & Desert Southwest \\
\hline Western Australia & East Pilbara, Shire of & Gulf Coast/Hawaii \\
\hline
\end{tabular}


Table 23.-Continued

\begin{tabular}{|c|c|c|}
\hline State & Municipality & U.S. Climate Region \\
\hline Western Australia & Esperance, Shire of & Desert Southwest \\
\hline Western Australia & Exmouth, Shire of & Gulf Coast/Hawaii \\
\hline Western Australia & Fremantle, City of & Desert Southwest \\
\hline Western Australia & Gingin, Shire of & Desert Southwest \\
\hline Western Australia & Gnowangerup, Shire of & Desert Southwest \\
\hline Western Australia & Goomalling, Shire of & Desert Southwest \\
\hline Western Australia & Gosnells, City of & Desert Southwest \\
\hline Western Australia & Greater Geraldton, City of & Desert Southwest \\
\hline Western Australia & Halls Creek, Shire of & Gulf Coast/Hawaii \\
\hline Western Australia & Harvey, Shire of & Desert Southwest \\
\hline Western Australia & Irwin, Shire of & Desert Southwest \\
\hline Western Australia & Jerramungup, Shire of & Desert Southwest \\
\hline Western Australia & Joondalup, City of & Desert Southwest \\
\hline Western Australia & Kalamunda, Shire of & Desert Southwest \\
\hline Western Australia & Kalgoorlie-Boulder, City of & Desert Southwest \\
\hline Western Australia & Katanning, Shire of & Desert Southwest \\
\hline Western Australia & Kellerberrin, Shire of & Desert Southwest \\
\hline Western Australia & Kent, Shire of & Desert Southwest \\
\hline Western Australia & Kojonup, Shire of & Desert Southwest \\
\hline Western Australia & Kondinin, Shire of & Desert Southwest \\
\hline Western Australia & Koorda, Shire of & Desert Southwest \\
\hline Western Australia & Kulin, Shire of & Desert Southwest \\
\hline Western Australia & Kwinana, Town of & Desert Southwest \\
\hline Western Australia & Lake Grace, Shire of & Desert Southwest \\
\hline Western Australia & Laverton, Shire of & Desert Southwest \\
\hline Western Australia & Leonora, Shire of & Desert Southwest \\
\hline Western Australia & Mandurah, City of & Desert Southwest \\
\hline Western Australia & Manjimup, Shire of & California Coast \\
\hline Western Australia & Meekatharra, Shire of & Gulf Coast/Hawaii \\
\hline Western Australia & Melville, City of & Desert Southwest \\
\hline Western Australia & Menzies, Shire of & Desert Southwest \\
\hline Western Australia & Merredin, Shire of & Desert Southwest \\
\hline Western Australia & Mingenew, Shire of & Desert Southwest \\
\hline Western Australia & Moora, Shire of & Desert Southwest \\
\hline Western Australia & Morawa, Shire of & Desert Southwest \\
\hline Western Australia & Mosman Park, Town of & Desert Southwest \\
\hline Western Australia & Mount Magnet, Shire of & Desert Southwest \\
\hline Western Australia & Mt Marshall, Shire of & Desert Southwest \\
\hline Western Australia & Mukinbudin, Shire of & Desert Southwest \\
\hline Western Australia & Mundaring, Shire of & Desert Southwest \\
\hline
\end{tabular}


Table 23.-Continued

\begin{tabular}{|c|c|c|}
\hline State & Municipality & U.S. Climate Region \\
\hline Western Australia & Murchison, Shire of & Desert Southwest \\
\hline Western Australia & Murray, Shire of & Desert Southwest \\
\hline Western Australia & Nannup, Shire of & Desert Southwest \\
\hline Western Australia & Narembeen, Shire of & Desert Southwest \\
\hline Western Australia & Narrogin, Shire of & Desert Southwest \\
\hline Western Australia & Narrogin, Town of & Desert Southwest \\
\hline Western Australia & Nedlands, City of & Desert Southwest \\
\hline Western Australia & Ngaanyatjarraku, Shire of & Desert Southwest \\
\hline Western Australia & Northam, Shire of & Desert Southwest \\
\hline Western Australia & Northampton, Shire of & Desert Southwest \\
\hline Western Australia & Nungarin, Shire of & Desert Southwest \\
\hline Western Australia & Peppermint Grove, Shire of & Desert Southwest \\
\hline Western Australia & Perenjori, Shire of & Desert Southwest \\
\hline Western Australia & Perth, City of & Desert Southwest \\
\hline Western Australia & Pingelly, Shire of & Desert Southwest \\
\hline Western Australia & Plantagenet, Shire of & California Coast \\
\hline Western Australia & Port Hedland, Town of & Gulf Coast/Hawaii \\
\hline Western Australia & Quairading, Shire of & Desert Southwest \\
\hline Western Australia & Ravensthorpe, Shire of & Desert Southwest \\
\hline Western Australia & Rockingham, City of & Desert Southwest \\
\hline Western Australia & Roebourne, Shire of & Gulf Coast/Hawaii \\
\hline Western Australia & Sandstone, Shire of & Desert Southwest \\
\hline Western Australia & Serpentine-Jarrahdale, Shire of & Desert Southwest \\
\hline Western Australia & Shark Bay, Shire of & Gulf Coast/Hawaii \\
\hline Western Australia & South Perth, City of & Desert Southwest \\
\hline Western Australia & Stirling, City of & Desert Southwest \\
\hline Western Australia & Subiaco, City of & Desert Southwest \\
\hline Western Australia & Swan, City of & Desert Southwest \\
\hline Western Australia & Tammin, Shire of & Desert Southwest \\
\hline Western Australia & Three Springs, Shire of & Desert Southwest \\
\hline Western Australia & Toodyay, Shire of & Desert Southwest \\
\hline Western Australia & Trayning, Shire of & Desert Southwest \\
\hline Western Australia & Upper Gascoyne, Shire of & Gulf Coast/Hawaii \\
\hline Western Australia & Victoria Park, Town of & Desert Southwest \\
\hline Western Australia & Victoria Plains, Shire of & Desert Southwest \\
\hline Western Australia & Vincent, Town of & Desert Southwest \\
\hline Western Australia & Wagin, Shire of & Desert Southwest \\
\hline Western Australia & Wandering, Shire of & Desert Southwest \\
\hline Western Australia & Wanneroo, City of & Desert Southwest \\
\hline Western Australia & Waroona, Shire of & Desert Southwest \\
\hline
\end{tabular}


Table 23.-Continued

\begin{tabular}{|l|l|l|}
\hline State & Municipality & U.S. Climate Region \\
\hline Western Australia & West Arthur, Shire of & Desert Southwest \\
\hline Western Australia & Westonia, Shire of & Desert Southwest \\
\hline Western Australia & Wickepin, Shire of & Desert Southwest \\
\hline Western Australia & Williams, Shire of & Desert Southwest \\
\hline Western Australia & Wiluna, Shire of & Desert Southwest \\
\hline Western Australia & Wongan - Ballidu, Shire of & Desert Southwest \\
\hline Western Australia & Woodanilling, Shire of & Desert Southwest \\
\hline Western Australia & Wyalkatchem, Shire of & Desert Southwest \\
\hline Western Australia & Wyndham-East Kimberley, Shire of & Gulf Coast/Hawaii \\
\hline Western Australia & Yalgoo, Shire of & Desert Southwest \\
\hline Western Australia & Yilgarn, Shire of & Desert Southwest \\
\hline Western Australia & York, Shire of & Desert Southwest \\
\hline
\end{tabular}


Table 24.-U.S. climate regions assigned to provinces in Canada

\begin{tabular}{|l|l|}
\hline Province & US Climate Region \\
\hline Alberta & Northern Tier \\
\hline British Columbia & Northern Tier \\
\hline Manitoba & Northern Tier \\
\hline New Brunswick & North Central \\
\hline Newfoundland and Labrador & Northern Tier \\
\hline Northwest Territories & Northern Tier \\
\hline Nova Scotia & North Central \\
\hline Nunavut & Northern Tier \\
\hline Ontario & North Central \\
\hline Prince Edward Island & North Central \\
\hline Quebec & North Central \\
\hline Saskatchewan & Northern Tier \\
\hline Yukon & Northern Tier \\
\hline
\end{tabular}

Table 25.-U.S. climate regions assigned to countries in Europe

\begin{tabular}{|l|l|}
\hline Country & U.S. Climate Zone \\
\hline Austria & Southeast \\
\hline Belgium & Pacific Northwest \\
\hline Bulgaria & Southwest \\
\hline Croatia & Southwest \\
\hline Cyprus & California Coast \\
\hline Czech Republic & Southeast \\
\hline Denmark & Pacific Northwest \\
\hline Germany & California Coast \\
\hline Greece & California Coast \\
\hline Estonia & Northern Tier \\
\hline Finland & Northern Tier \\
\hline France & California Coast \\
\hline Hungary & Southeast \\
\hline Iceland & Northern Tier \\
\hline Ireland & Pacific Northwest \\
\hline Italy & California Coast \\
\hline Latvia & Northern Tier \\
\hline Liechtenstein & Southeast \\
\hline Lithuania & Northern Tier \\
\hline Luxembourg & Pacific Northwest \\
\hline Malta & California Coast \\
\hline Montenegro & California Coast \\
\hline Netherlands & Pacific Northwest \\
\hline & \\
\hline
\end{tabular}




\begin{tabular}{|l|l|}
\hline Country & U.S. Climate Zone \\
\hline Norway & Northern Tier \\
\hline Poland & Pacific Northwest \\
\hline Portugal & California Coast \\
\hline Republic of Macedonia & California Coast \\
\hline Romania & Southwest \\
\hline Spain & California Coast \\
\hline Slovakia & Southwest \\
\hline Slovenia & Southwest \\
\hline Switzerland & Southeast \\
\hline Sweden & Northern Tier \\
\hline Turkey & Pacific Northwest \\
\hline United Kingdom & Pacific Northwest \\
\hline $\begin{array}{l}\text { Note: } \text { not all countries in Europe are assessed in i-Tree due to limited } \\
\text { data for the missing countries. }\end{array}$ & \\
\hline
\end{tabular}


Table 26.-U.S. climate regions assigned to municipalities in Mexico

\begin{tabular}{|c|c|c|}
\hline State & Municipality & US Climate Region \\
\hline Aguascalientes & Aguascalientes & California Coast \\
\hline Baja California & Mexicali & Desert Southwest \\
\hline Baja California & Playas de Rosarito & California Coast \\
\hline Baja California & Tecate & California Coast \\
\hline Baja California & Tijuana & California Coast \\
\hline Baja California Sur & La Paz & Gulf Coast/Hawaii \\
\hline Campeche & Campeche & Gulf Coast/Hawaii \\
\hline Chiapas & Tuxtla Gutierrez & Gulf Coast/Hawaii \\
\hline Chihuahua & Aldama & South Central \\
\hline Chihuahua & Aquiles Serdan & South Central \\
\hline Chihuahua & Chihuahua & South Central \\
\hline Chihuahua & Juarez & South Central \\
\hline Ciudad de Mexico & Alvaro Obregon & California Coast \\
\hline Ciudad de Mexico & Azcapotzalco & California Coast \\
\hline Ciudad de Mexico & Benito Juarez & California Coast \\
\hline Ciudad de Mexico & Coyoacan & California Coast \\
\hline Ciudad de Mexico & Cuajimalpa de Morelos & California Coast \\
\hline Ciudad de Mexico & Cuauhtemoc & California Coast \\
\hline Ciudad de Mexico & Gustavo A. Madero & California Coast \\
\hline Ciudad de Mexico & Iztacalco & California Coast \\
\hline Ciudad de Mexico & Iztapalapa & California Coast \\
\hline Ciudad de Mexico & Magdalena Contreras & California Coast \\
\hline Ciudad de Mexico & Miguel Hidalgo & California Coast \\
\hline Ciudad de Mexico & Milpa Alta & California Coast \\
\hline Ciudad de Mexico & Tlahuac & California Coast \\
\hline Ciudad de Mexico & Tlalpan & California Coast \\
\hline Ciudad de Mexico & Venustiano Carranza & California Coast \\
\hline Ciudad de Mexico & Xochimilco & California Coast \\
\hline Coahuila & Matamoros & Desert Southwest \\
\hline Coahuila & Torreon & Desert Southwest \\
\hline Colima & Colima & Gulf Coast/Hawaii \\
\hline Durango & Durango & Desert Southwest \\
\hline Durango & Gomez Palacio & Desert Southwest \\
\hline Durango & Lerdo & Desert Southwest \\
\hline Estado de Mexico & Acolman & California Coast \\
\hline Estado de Mexico & Almoloya de Juarez & Southeast \\
\hline Estado de Mexico & Amecameca & California Coast \\
\hline Estado de Mexico & Apaxco & California Coast \\
\hline Estado de Mexico & Atenco & California Coast \\
\hline
\end{tabular}


Table 26._Continued

\begin{tabular}{|c|c|c|}
\hline State & Municipality & US Climate Region \\
\hline Estado de Mexico & Atizapan de Zaragoza & California Coast \\
\hline Estado de Mexico & Atlautla & California Coast \\
\hline Estado de Mexico & Axapusco & California Coast \\
\hline Estado de Mexico & Ayapango & California Coast \\
\hline Estado de Mexico & Calimaya & Southeast \\
\hline Estado de Mexico & Chalco & California Coast \\
\hline Estado de Mexico & Chapultepec & Southeast \\
\hline Estado de Mexico & Chiautla & California Coast \\
\hline Estado de Mexico & Chicoloapan & California Coast \\
\hline Estado de Mexico & Chiconcuac & California Coast \\
\hline Estado de Mexico & Chimalhuacan & California Coast \\
\hline Estado de Mexico & Coacalco de Berriozabal & California Coast \\
\hline Estado de Mexico & Cocotitlan & California Coast \\
\hline Estado de Mexico & Coyotepec & California Coast \\
\hline Estado de Mexico & Cuautitlan & California Coast \\
\hline Estado de Mexico & Cuautitlan Izcalli & California Coast \\
\hline Estado de Mexico & Ecatepec de Morelos & California Coast \\
\hline Estado de Mexico & Ecatzingo & California Coast \\
\hline Estado de Mexico & Huehuetoca & California Coast \\
\hline Estado de Mexico & Hueypoxtla & California Coast \\
\hline Estado de Mexico & Huixquilucan & California Coast \\
\hline Estado de Mexico & Isidro Fabela & California Coast \\
\hline Estado de Mexico & Ixtapaluca & California Coast \\
\hline Estado de Mexico & Jaltenco & California Coast \\
\hline Estado de Mexico & Jilotzingo & California Coast \\
\hline Estado de Mexico & Juchitepec & California Coast \\
\hline Estado de Mexico & La Paz & California Coast \\
\hline Estado de Mexico & Lerma & Southeast \\
\hline Estado de Mexico & Melchor Ocampo & California Coast \\
\hline Estado de Mexico & Metepec & Southeast \\
\hline Estado de Mexico & Mexicaltzingo & Southeast \\
\hline Estado de Mexico & Naucalpan de Juarez & California Coast \\
\hline Estado de Mexico & Nextlalpan & California Coast \\
\hline Estado de Mexico & Nezahualcoyotl & California Coast \\
\hline Estado de Mexico & Nicolas Romero & California Coast \\
\hline Estado de Mexico & Nopaltepec & California Coast \\
\hline Estado de Mexico & Ocoyoacac & Southeast \\
\hline Estado de Mexico & Otumba & California Coast \\
\hline Estado de Mexico & Otzolotepec & Southeast \\
\hline
\end{tabular}


Table 26._Continued

\begin{tabular}{|c|c|c|}
\hline State & Municipality & US Climate Region \\
\hline Estado de Mexico & Ozumba & California Coast \\
\hline Estado de Mexico & Papalotla & California Coast \\
\hline Estado de Mexico & Rayon & Southeast \\
\hline Estado de Mexico & San Antonio la Isla & Southeast \\
\hline Estado de Mexico & San Martin de las Piramides & California Coast \\
\hline Estado de Mexico & San Mateo Atenco & Southeast \\
\hline Estado de Mexico & Tecamac & California Coast \\
\hline Estado de Mexico & Temamatla & California Coast \\
\hline Estado de Mexico & Temascalapa & California Coast \\
\hline Estado de Mexico & Temoaya & Southeast \\
\hline Estado de Mexico & Tenango del Aire & California Coast \\
\hline Estado de Mexico & Tenango del Valle & California Coast \\
\hline Estado de Mexico & Teoloyucan & California Coast \\
\hline Estado de Mexico & Teotihuacan & California Coast \\
\hline Estado de Mexico & Tepetlaoxtoc & California Coast \\
\hline Estado de Mexico & Tepetlixpa & California Coast \\
\hline Estado de Mexico & Tepotzotlan & California Coast \\
\hline Estado de Mexico & Tequixquiac & California Coast \\
\hline Estado de Mexico & Texcoco & California Coast \\
\hline Estado de Mexico & Tezoyuca & California Coast \\
\hline Estado de Mexico & Tlalmanalco & California Coast \\
\hline Estado de Mexico & Tlalnepantla de Baz & California Coast \\
\hline Estado de Mexico & Toluca & Southeast \\
\hline Estado de Mexico & Tonanitla & California Coast \\
\hline Estado de Mexico & Tultepec & California Coast \\
\hline Estado de Mexico & Tultitlan & California Coast \\
\hline Estado de Mexico & Valle de Chalco Solidaridad & California Coast \\
\hline Estado de Mexico & Villa del Carbon & California Coast \\
\hline Estado de Mexico & Xonacatlan & Southeast \\
\hline Estado de Mexico & Zinacantepec & Southeast \\
\hline Estado de Mexico & Zumpango & California Coast \\
\hline Guanajuato & Irapuato & Southwest \\
\hline Guanajuato & Leon & Southwest \\
\hline Guanajuato & Silao de la Victoria & Southwest \\
\hline Guerrero & Acapulco de Juarez & Gulf Coast/Hawaii \\
\hline Guerrero & Coyuca de Benitez & Gulf Coast/Hawaii \\
\hline Hidalgo & Pachuca de Soto & California Coast \\
\hline Hidalgo & Tizayuca & California Coast \\
\hline Jalisco & El Salto & Gulf Coast/Hawaii \\
\hline
\end{tabular}


Table 26.-Continued

\begin{tabular}{|c|c|c|}
\hline State & Municipality & US Climate Region \\
\hline Jalisco & Guadalajara & Gulf Coast/Hawaii \\
\hline Jalisco & Ixtlahuacan de los Membrillos & Gulf Coast/Hawaii \\
\hline Jalisco & Juanacatlan & Gulf Coast/Hawaii \\
\hline Jalisco & Tlajomulco de Zuniga & Gulf Coast/Hawaii \\
\hline Jalisco & Tlaquepaque & Gulf Coast/Hawaii \\
\hline Jalisco & Tonala & Gulf Coast/Hawaii \\
\hline Jalisco & Zapopan & Gulf Coast/Hawaii \\
\hline Michoacan & Charo & California Coast \\
\hline Michoacan & Morelia & California Coast \\
\hline Michoacan & Tarimbaro & California Coast \\
\hline Morelos & Cuernavaca & Gulf Coast/Hawaii \\
\hline Nayarit & Tepic & Gulf Coast/Hawaii \\
\hline Nayarit & Xalisco & Gulf Coast/Hawaii \\
\hline Nuevo Leon & Apodaca & Desert Southwest \\
\hline Nuevo Leon & Cadereyta Jimenez & Desert Southwest \\
\hline Nuevo Leon & Carmen & Desert Southwest \\
\hline Nuevo Leon & Garcia & Desert Southwest \\
\hline Nuevo Leon & General Escobedo & Desert Southwest \\
\hline Nuevo Leon & Guadalupe & Desert Southwest \\
\hline Nuevo Leon & Juarez & Desert Southwest \\
\hline Nuevo Leon & Monterrey & Desert Southwest \\
\hline Nuevo Leon & Salinas Victoria & Desert Southwest \\
\hline Nuevo Leon & San Nicolas de los Garza & Desert Southwest \\
\hline Nuevo Leon & San Pedro Garza Garcia & Desert Southwest \\
\hline Nuevo Leon & Santa Catarina & Desert Southwest \\
\hline Nuevo Leon & Santiago & Desert Southwest \\
\hline Oaxaca & Animas Trujano & Gulf Coast/Hawaii \\
\hline Oaxaca & Oaxaca de Juarez & Gulf Coast/Hawaii \\
\hline Oaxaca & San Agustin de las Juntas & Gulf Coast/Hawaii \\
\hline Oaxaca & San Agustin Yatareni & Gulf Coast/Hawaii \\
\hline Oaxaca & San Andres Huayapam & Gulf Coast/Hawaii \\
\hline Oaxaca & San Antonio de la Cal & Gulf Coast/Hawaii \\
\hline Oaxaca & San Bartolo Coyotepec & Gulf Coast/Hawaii \\
\hline Oaxaca & San Jacinto Amilpas & Gulf Coast/Hawaii \\
\hline Oaxaca & San Lorenzo Cacaotepec & Gulf Coast/Hawaii \\
\hline Oaxaca & San Pablo Etla & Gulf Coast/Hawaii \\
\hline Oaxaca & San Sebastian Tutla & Gulf Coast/Hawaii \\
\hline Oaxaca & Santa Cruz Amilpas & Gulf Coast/Hawaii \\
\hline Oaxaca & Santa Cruz Xoxocotlan & Gulf Coast/Hawaii \\
\hline
\end{tabular}


Table 26.-Continued

\begin{tabular}{|c|c|c|}
\hline State & Municipality & US Climate Region \\
\hline Oaxaca & Santa Lucia del Camino & Gulf Coast/Hawaii \\
\hline Oaxaca & Santa Maria Atzompa & Gulf Coast/Hawaii \\
\hline Oaxaca & Santa Maria Coyotepec & Gulf Coast/Hawaii \\
\hline Oaxaca & Santa Maria del Tule & Gulf Coast/Hawaii \\
\hline Oaxaca & Santo Domingo Tomaltepec & Gulf Coast/Hawaii \\
\hline Oaxaca & Soledad Etla & Gulf Coast/Hawaii \\
\hline Oaxaca & Tlalixtac de Cabrera & Gulf Coast/Hawaii \\
\hline Oaxaca & Villa de Etla & Gulf Coast/Hawaii \\
\hline Oaxaca & Villa de Zaachila & Gulf Coast/Hawaii \\
\hline Puebla & Acajete & California Coast \\
\hline Puebla & Amozoc & California Coast \\
\hline Puebla & Chiautzingo & California Coast \\
\hline Puebla & Coronango & California Coast \\
\hline Puebla & Cuautlancingo & California Coast \\
\hline Puebla & Domingo Arenas & California Coast \\
\hline Puebla & Huejotzingo & California Coast \\
\hline Puebla & Juan C. Bonilla & California Coast \\
\hline Puebla & Ocoyucan & California Coast \\
\hline Puebla & Puebla & California Coast \\
\hline Puebla & San Andres Cholula & California Coast \\
\hline Puebla & San Felipe Teotlalcingo & California Coast \\
\hline Puebla & San Gregorio Atzompa & California Coast \\
\hline Puebla & San Martin Texmelucan & California Coast \\
\hline Puebla & San Miguel Xoxtla & California Coast \\
\hline Puebla & San Pedro Cholula & California Coast \\
\hline Puebla & San Salvador el Verde & California Coast \\
\hline Puebla & Tepatlaxco de Hidalgo & California Coast \\
\hline Puebla & Tlaltenango & California Coast \\
\hline Queretaro & Corregidora & California Coast \\
\hline Queretaro & El Marques & California Coast \\
\hline Queretaro & Huimilpan & California Coast \\
\hline Queretaro & Queretaro & California Coast \\
\hline Quintana Roo & Benito Juarez & Gulf Coast/Hawaii \\
\hline San Luis Potosi & San Luis Potosi & Southwest \\
\hline San Luis Potosi & Soledad de Graciano Sanchez & Southwest \\
\hline Sinaloa & Culiacan & Gulf Coast/Hawaii \\
\hline Sonora & Guaymas & Gulf Coast/Hawaii \\
\hline Tabasco & Centro (Villahermosa) & Gulf Coast/Hawaii \\
\hline Tamaulipas & Tampico & Gulf Coast/Hawaii \\
\hline
\end{tabular}


Table 26.-Continued

\begin{tabular}{|c|c|c|}
\hline State & Municipality & US Climate Region \\
\hline Tlaxcala & Acuamanala de Miguel Hidalgo & California Coast \\
\hline Tlaxcala & Ixtacuixtla de Mariano Matamoros & California Coast \\
\hline Tlaxcala & Mazatecochco de Jose Maria Morelos & California Coast \\
\hline Tlaxcala & Nativitas & California Coast \\
\hline Tlaxcala & Papalotla de Xicohtencatl & California Coast \\
\hline Tlaxcala & San Jeronimo Zacualpan & California Coast \\
\hline Tlaxcala & San Juan Huactzinco & California Coast \\
\hline Tlaxcala & San Lorenzo Axocomanitla & California Coast \\
\hline Tlaxcala & San Pablo del Monte & California Coast \\
\hline Tlaxcala & Santa Ana Nopalucan & California Coast \\
\hline Tlaxcala & Santa Apolonia Teacalco & California Coast \\
\hline Tlaxcala & Santa Catarina Ayometla & California Coast \\
\hline Tlaxcala & Santa Cruz Quilehtla & California Coast \\
\hline Tlaxcala & Tenancingo & California Coast \\
\hline Tlaxcala & Teolocholco & California Coast \\
\hline Tlaxcala & Tepetitla de Lardizabal & California Coast \\
\hline Tlaxcala & Tepeyanco & California Coast \\
\hline Tlaxcala & Tetlatlahuca & California Coast \\
\hline Tlaxcala & Xicohtzinco & California Coast \\
\hline Tlaxcala & Zacatelco & California Coast \\
\hline Veracruz & Banderilla & Gulf Coast/Hawaii \\
\hline Veracruz & Coatepec & Gulf Coast/Hawaii \\
\hline Veracruz & Emiliano Zapata & Gulf Coast/Hawaii \\
\hline Veracruz & Jilotepec & Gulf Coast/Hawaii \\
\hline Veracruz & Rafael Lucio & Gulf Coast/Hawaii \\
\hline Veracruz & Tlalnelhuayocan & Gulf Coast/Hawaii \\
\hline Veracruz & Xalapa & Gulf Coast/Hawaii \\
\hline Yucatan & Conkal & Gulf Coast/Hawaii \\
\hline Yucatan & Kanasin & Gulf Coast/Hawaii \\
\hline Yucatan & Merida & Gulf Coast/Hawaii \\
\hline Yucatan & Ucu & Gulf Coast/Hawaii \\
\hline Yucatan & Uman & Gulf Coast/Hawaii \\
\hline Zacatecas & Zacatecas & Southwest \\
\hline \multicolumn{3}{|c|}{$\begin{array}{l}\text { Note: The procedures of estimating tree effects on energy use was developed based on average U.S. } \\
\text { building types and energy uses within U.S. climate regions. Applications of this process outside of } \\
\text { the United States will produce results that assume that the U.S. conditions (e.g., climate, building } \\
\text { types, heating fuel usage) apply to the foreign location. The only local values used in the estimates for } \\
\text { Australia, Canada, Europe, and Mexico are electricity and fuel costs. }\end{array}$} \\
\hline
\end{tabular}

All other variables used in the energy calculations are based on the formulas and tables in McPherson and Simpson 1999. 